\title{
Endovascular coil migration and upper gastro- intestinal bleed: a causal or casual relationship?
}

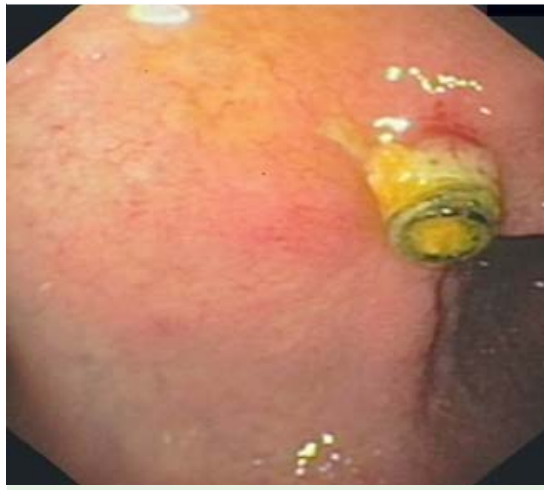

Fig. 1 Endoscopic image of the coil in the duodenum 2 years before the present admission.

A 77-year-old man was admitted with an acute cerebral infarct for which he was started on low-molecular-weight heparin and antiplatelets on admission. He had no history of hematemesis or melena; however, his nasogastric tube showed the presence of altered blood.

He had a significant history of massive hematemesis 4 years previously, for which he underwent emergency coil embolization of the gastroduodenal artery following a failed attempt at endoscopic hemostasis. He was readmitted 2 years later with a pontine infarct, and an esophagogastroduodenoscopy (EGD) showed protrusion of the coil into the duodenum
( $\bullet$ Fig. 1). A contrast computed tomography (CT) scan of the abdomen showed metallic coils occluding the gastroduodenal artery, and a portion of the coil projected focally and endoluminally through the medial wall of the first part of the duodenum ( $\bullet$ Fig. 2).

During the present admission, an EGD showed a deformed coil protruding through the anterior wall of the duodenum, and an adjacent clean-based ulcer $<0.5 \mathrm{~cm}$ in size ( Fig.3). Abdominal radiograph showed the presence of two coils in the abdomen, one of which was partially deformed ( $\bullet$ Fig.4). The patient was managed conservatively with intravenous proton pump inhibitors. As he had no overt gastrointestinal bleeding or drop in hemoglobin level, an angiography or contrast CT was not performed.

When endoscopy and medical management fails, interventional embolotherapy is not only a good alternative to surgery but is now considered to be the therapy of choice [1]. Migration of the coil is a very rare but known complication, with some coils even being passed per rectum [2-5]. Fortunately in this case, the migration was only local, self-limiting, and apparently nonprogressive. It is unclear, however, whether the ulcer and bleed were caused by the coil or were due to the use of antiplatelet drugs. Patients undergoing coil embolization should therefore be carefully followed up periodically for any such delayed complications.

Endoscopy_UCTN_Code_CPL_1AK_2AI

Competing interests: None

Naveen Mohandas, Mukundan Swaminathan, Vijayanand Vegiraju, K. V. K. S. N. Murthy, Aditya Kulkarni, Venkatakrishnan Leelakrishnan, Krishnaveni Janarthan

Department of Gastroenterology, PSG Institute of Medical Science and Research, Peelamedu, Coimbatore, Tamil Nadu, India

\section{References}

1 Loffroy RF, Abualsaud BA, Lin MD et al. Recent advances in endovascular techniques for management of acute nonvariceal upper gastrointestinal bleeding. World J Gastrointest Surg 2011; 3: 89-100

2 Jaurigue MM, Snyder M, Cannon M. Recurrent upper GI bleeding secondary to coil migration in a patient with known NSAIDinduced peptic ulcer disease. Gastrointest Endosc 2014; 79: 1004; discussion 1005

3 Singh G, Denyer M, Patel JV. Endoscopic visualization of embolization coil in a duodenal ulcer. Gastrointest Endosc 2008; 67: $351-352$

4 Kuhara A, Koganemaru M, Ishikawa $\mathrm{H}$ et al. Late-occurring coil migration into the duodenum. BMJ Case Rep 2013. DOI: 10.1136/ bcr-2012-007759

5 Skipworth JR, Morkane C, Raptis DA et al. Coil migration - a rare complication of endovascular exclusion of visceral artery pseudoaneurysms and aneurysms. Ann R Coll Surg Engl 2011; 93: e19-e23

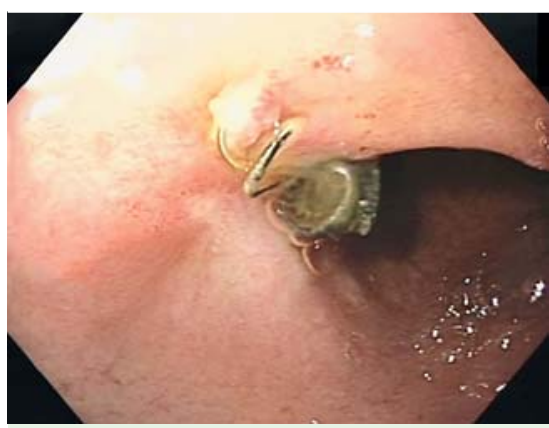

Fig. 3 Esophagogastroduodenoscopy during the present admission, showing the deformed and protruding coil with ulcer in the duodenum. 


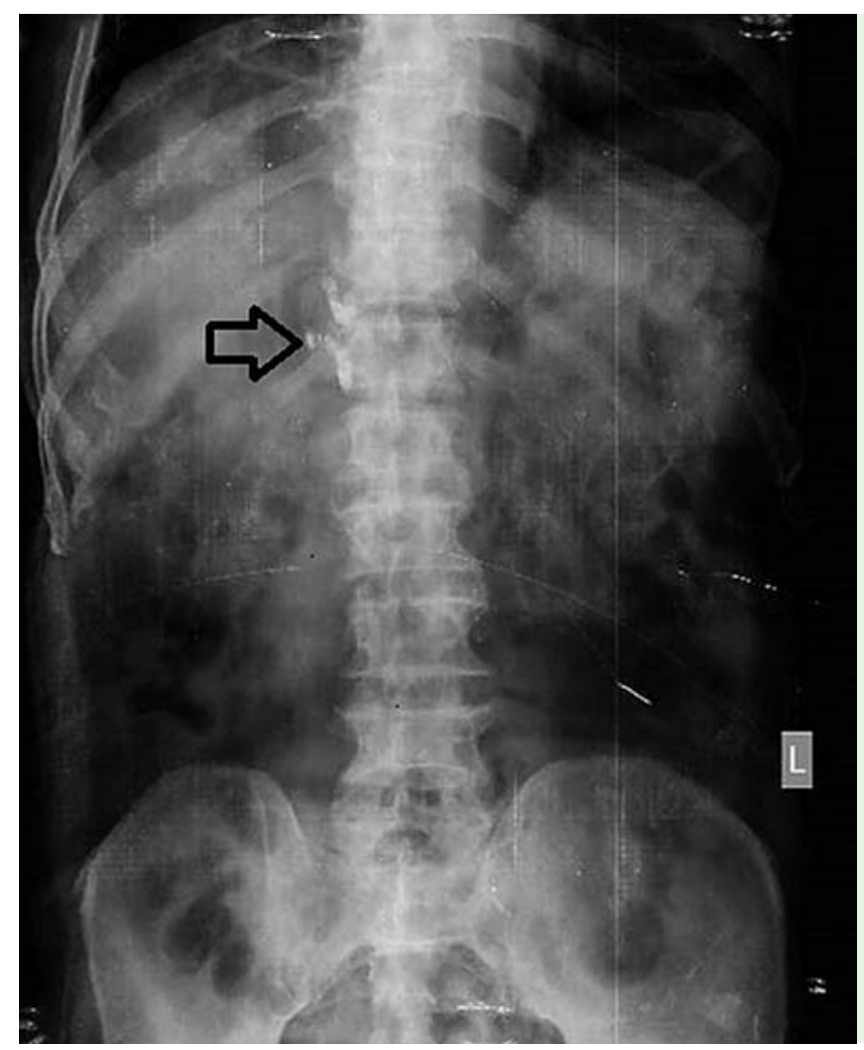

Fig. 4 Abdominal radiograph during the present admission, showing two coils, one of which was deformed (black arrow).

\section{Bibliography}

Dol http://dx.doi.org/

10.1055/s-0034-1392505

Endoscopy 2015; 47: E389-E390

(c) Georg Thieme Verlag KG

Stuttgart · New York

ISSN 0013-726X

\section{Corresponding author} Naveen Mohandas, MD, MRCP(UK), DM

Department of Gastroenterology

PSG Institute of Medical Science and Research Peelamedu, Coimbatore

Tamil Nadu

India 641004

Fax: +91-42-22594400

naveenmd212@gmail.com 